\title{
Polarization puzzles for the upper elementary grades
}

\section{Meera Chandrasekhar, David Rainwater, Rebecca Litherland, Rodney Swope, Ann VanNest}

Meera Chandrasekhar, David L. Rainwater, Rebecca Q. Litherland, Rodney A. Swope, Ann VanNest, "Polarization puzzles for the upper elementary grades," Proc. SPIE 2525, 1995 International Conference on Education in Optics, (13 October 1995); doi: 10.1117/12.224011

Event: SPIE's 1995 International Symposium on Optical Science, Engineering, and Instrumentation, 1995, San Diego, CA, United States 


\title{
Polarization Puzzles for the Upper Elementary Grades
}

\author{
Meera Chandrasekhar ${ }^{+}$and David L. Rainwater* \\ Department of Physics \\ University of Missouri, Columbia Mo 65211 \\ Rebecca Q. Litherland, Rodney A. Swope and Ann VanNest \\ Science Department, Columbia Public Schools \\ 1206 E. Walnut Street, Columbia MO 65201
}

\begin{abstract}
The concept of polarization and its most basic consequence, Malus' Law, is usually not taught in the elementary or middle grades because of conceptual difficulties. We describe a series of puzzles to teach these concepts. We introduce the concept of polarization using sunglasses to understand the consequences of parallel and crossed polarizers. We then expand the concept with four puzzles. The puzzles are cut out of sheets of linear polarizers and are viewed through a (hand held) spinning polarizer. The first puzzle is constructed out of wedge shaped pieces of linear polarizer so that the wheel appears to rotate when viewed through the spinning polarizer. The second puzzle consists of concentric circles that appear to radiate outward. The third and fourth puzzles are four- and twelve piece wedges that are manipulated to produce different symmetric designs.

We have tested these activities on fifth and sixth graders, and find that they enjoy the manipulative as well as the problem solving aspects of the puzzles. They are also able to understand that when light is polarized, "whatever it is that waves" (the electric field) is oriented in one direction. The materials are inexpensive and can be easily made by teachers for classroom learning.
\end{abstract}

Keywords: linear polarizers, puzzles, Malus' Law, elementary and middle schools.

\section{INTRODUCTION}

The polarization of light is a phenomenon that is typically rather difficult for young students to understand, yet it is a consequence of one of the most fundamental differences between light and sound waves. While several features of the wave nature of light and sound can be illustrated in classic experiments such as Young's double slit experiment, it is the transverse nature of light makes polarization possible, while polarization is not possible for longitudinal sound waves.

In this paper we will discuss several experiments and games based on the polarization of light. These activities have been tested on fifth and sixth grade students. The students have found all the activities interesting. The first two activities are well known. We include them in this paper for the 
sake of completeness. After the initial discovery phase of the activity, we have found that students are able to successfully predict the answers to the later puzzles. The last puzzle has been the most challenging of the series, but can be understood well by a large fraction of the students.

\section{WHAT IS POLARIZATION?}

In the first activity we investigate what polarization means. We start with a demonstration for the class.

Materials: Thick rope or slinky.

1. Two students hold opposite ends of the rope. One of them wiggles the rope to make a wave.

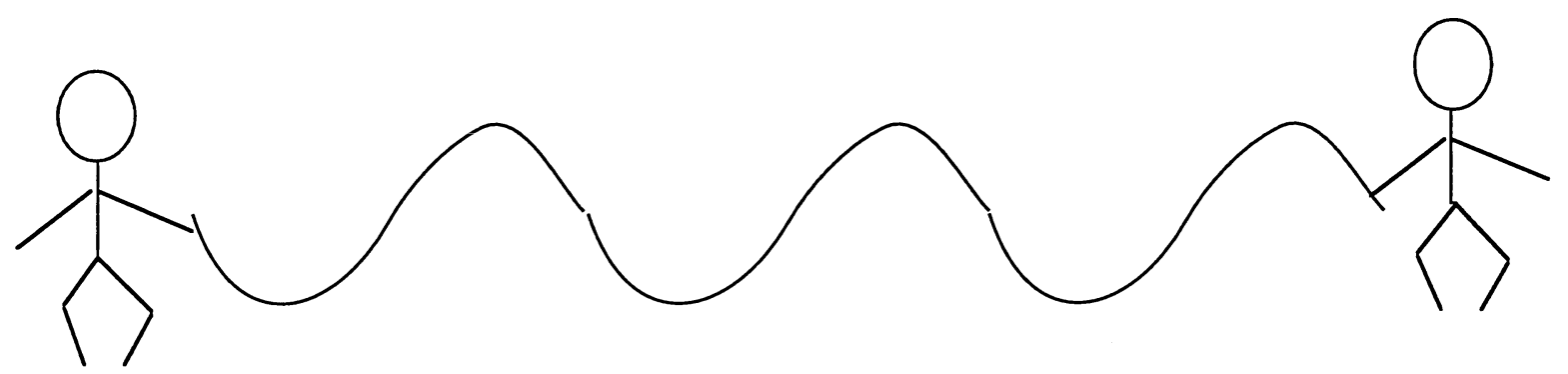

2. The rope is wiggled in different directions so that the wiggles are vertical, horizontal, or at an angle. Students are asked to notice how the wave travels from one person to the other, but that the wiggles point at right angles to the direction in which the wave travels, regardless of whether the rope is wiggled up-down, left-right or at an angle. A wave where the wiggles are at right angles to the direction of travel of the wave is described as a transverse wave.

At this stage longitudinal waves are introduced, and this is best done with a slinky. A compressional wave is sent down the slinky, and students are asked to notice how the "wiggle" in the wave now is along the direction of travel of the wave, rather than at $90^{\circ}$ as in the transverse wave.

What wiggles to produce light? In a light wave, it is electric fields that wiggle ${ }^{1}$. Light is a transverse wave. In ordinary light, the wiggles point in all possible directions that are at right angles to the direction in which light travels (up-down, left-right, etc.). If a beam of light coming from a lamp is blocked with a sheet of paper, the wiggles point in all possible directions on that sheet of paper. 

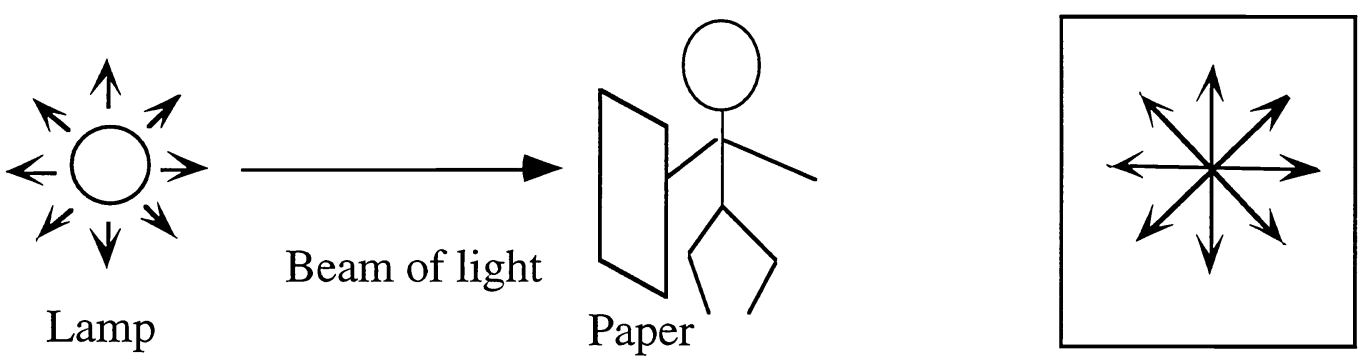

The wiggles are in all directions on this sheet of paper.

The discussion is then led to the question, "What is polarization?" Polarization means that one of those many directions in which the wave wiggles has been picked out. When the rope is wiggled in one direction, the wave is polarized because the wiggles point in only one direction (up - down, or left - right, for example). When light wiggles in all directions, it is called unpolarized. This means it has no special direction for the wiggles.

How would one make polarized light? One of the ways is to send the unpolarized light through a polarizer. The next step discusses how a polarizer works.

3. Lay the rope straight on the floor. Line up all but two of the students and have them stand with their feet on either side of the rope, about 6 inches apart. Have two students hold the ends of the rope and wiggle it up and down.

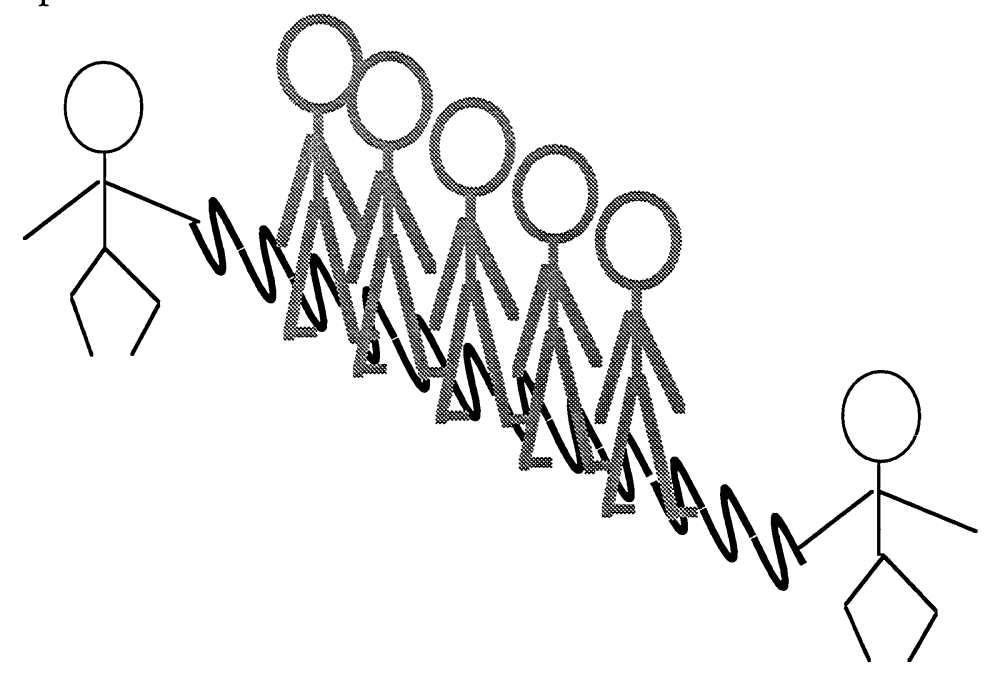

Students then answer the following questions:

Does the wave with wiggles going up and down go through their legs? (Answer: yes, it does.)

Now try waving the rope sideways. Does the wave go through their legs?

(Answer: no, or only with difficulty) 
Why did the rope wave do what it did?

(Answer: The legs of the students acted like a set of slats (like a cake rack or picket fence). When the rope waved along the long direction in the slats (legs), it went through. When it waved across, it did not go through. This is a polarizer for the "rope wave".) ${ }^{2}$

For light, where the wavelengths are much smaller, the slats of the polarizer have to be very close together. A polarizer is made by putting long molecules into a plastic and heat treating them so they line up. When a polarizer is placed over regular (unpolarized) light, only one direction of wiggles can come through the polarizer.

We now send a compressional (longitudinal) wave using the slinky through the legs of the students. It always gets through, and there is no way to choose one direction of wiggles since the direction of travel is the same as the direction of the wiggles. We discuss how there are no preferred directions for a longitudinal wave, and how one cannot polarize it.

\section{SUNGLASSES AND POLARIZERS}

In the next set of activities, the students investigate the properties of linearly polarized light.

Materials: Two pairs of polarized sunglasses, squares of polarizers with their transmission axes marked, and a light box.

1. Lay one of the polarizers on the light box,with its arrow pointing up.

How much light goes through (none, a lot, a little)? (Answer: a lot)

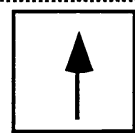

2. Rotate the polarizer.

How much light goes through (none, a lot, a little)? (Answer: a lot)

How does it compare to the light that went through in step 1?(Answer: the same)

Why is the amount of light going through what it is? (The students may not have an answer to this question at this point. They should be able to answer it better after they have completed the activity. Answer: the light coming from the light box is not polarized, so changing the angle of the polarizer will not change the amount of light coming through.)

3 The students now proceed to activities where they overlay lay two polarizers. The first polarizer produces the polarized light. By varying the angle of the transmission axis of the second polarizer relative to the first, the amount of polarized light going through is varied. 
Lay one polarizer over the other so the arrows point as shown in the table below. At each stage students predict the amount of light going through before they try the experiment.

How much light goes through?

\begin{tabular}{|c|c|c|c|}
\hline & Prediction: & Now try it: & Answer \\
\hline & & & $\begin{array}{l}\text { A lot of light goes through: the } \\
\text { polarizers look light gray. }\end{array}$ \\
\hline & & & $\begin{array}{l}\text { No light goes through: the } \\
\text { polarizers look black. }\end{array}$ \\
\hline & & & $\begin{array}{l}\text { Some light goes through: the } \\
\text { polarizers look dark gray. }\end{array}$ \\
\hline
\end{tabular}

4. The students then perform the same experiments with polarized sunglasses. We have found that the level of interest surges when they find that sunglasses behave the same way.

How much light goes through?

Prediction:
Now try it:

Primary concept learned: If light is polarized vertical, it will go through another polarizer that allows vertically polarized light to go through, but will not go through a polarizer that allows horizontally polarized light to go through. This is like having two sets of picket fences, with their slats pointing at right angles to each other. If the polarizer is at an angle in between, some of the light will go through. This is, of course, Malus' Law in qualitative form. 
The ability to control light by rotating a polarizer is possible only if one has polarized light to begin with. In these activities, polarized light was produced by the first polarizer, and the light coming through was then controlled using the second polarizer. If one has only unpolarized light as in steps 1 and 2, rotating the polarizer does not change the intensity of the light.

We then discuss the question "Why, then, do polarized sunglasses work?" Because sunlight is scattered by the molecules in the atmosphere, and becomes partially polarized. The transmission axis of sunglasses are set so that they are perpendicular to the usual polarization of sunlight. If, instead, one rotated the sunglasses by $90^{\circ}$, they would not work quite as effectively.

In the next two activities we use the rotation of the transmission axis of polarizers to produce wheel-like designs. For most of these activities we use a spinner. A spinner can be purchased from companies that sell polarizer sheets or can be home made. Ours are made of 8 " circles cut from 1/32" thick linear polarizer sheets. The center of the circle is nailed to a 12 " long dowel rod used as a handle.

\section{THE SPINNING WHEEL}

Materials: Polarizers cut in wedges as described below, polarizing spinner or polarized sunglasses, light box.

Triangular wedges are cut from a strip of linear polarizer in the pattern shown below. The arrow in the pattern indicates the direction of the transmission axis. The wedges are cut ahead of class time, so that the students do not know how the pieces were cut.

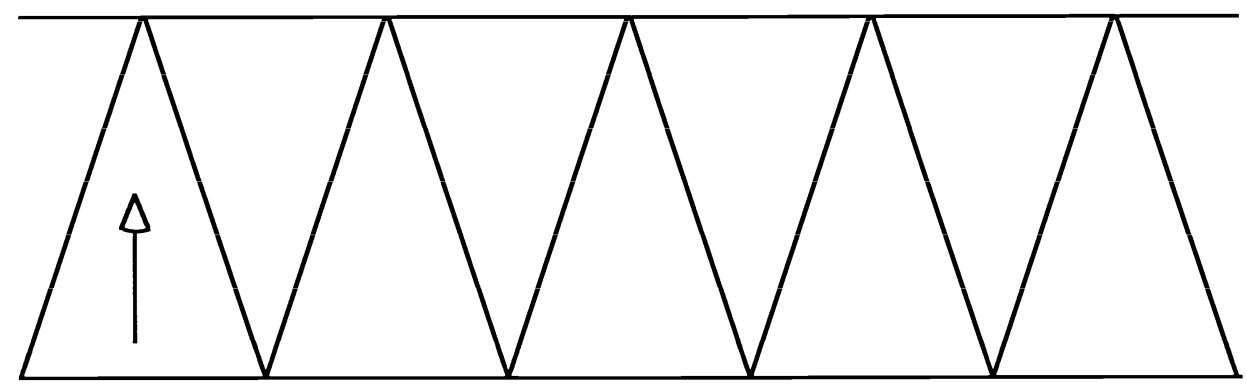

1. The students are asked to assemble the triangular pieces into a circle. They are asked to look at the circle through their spinners, rotate their spinners and asked what they see. (Answer: they should see a spinning wheel.)

2. They are then asked how are axes of the polarizer are oriented relative to each other. (Answer: the axes are radial).

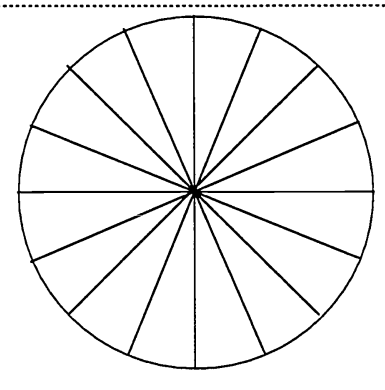


If they do not get the answer to the previous question, or to double check their answer, they are asked to take the wheel apart and lay the pieces in a straight line (with the points of the wedges pointing up) and look through the spinner. All the pieces should look the same color.

\section{THE FLASHING WHEEL}

Materials: Polarizer spinner or sunglasses, light box, and wheels cut in concentric circles as shown below.

For this activity, six concentric circles are cut from a single six inch circle of linear polarizer as shown in the figure. It is convenient to cut the smallest circle one inch in diameter, and the other circles in one inch increments.

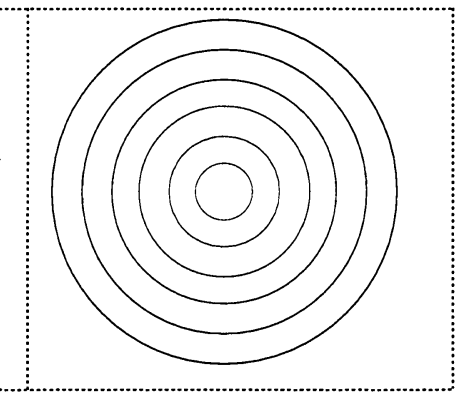

1. The students are first asked to assemble the pieces so the whole circle is a single color.

2. The students then rotate the pieces so they get a pattern that radiates from the center outward as they turn the spinner.

3. They are then asked to draw the relative orientation of the transmission axes of the circles in step 2.

\section{SUNGLASS PUZZLES}

The next two activities are puzzles made of four and twelve pieces. These puzzles may be viewed either through a pair of sunglasses or through a stationary spinner.

\subsection{The four piece puzzle}

The four piece puzzle is made by cutting quadrants in a circle of $1 / 32$ " thick linear polarizer. Again, students are not shown how the pieces were cut, but are just handed the pieces. They are then lead through a series of steps where they have to use their knowledge of Malus' Law (in qualitative form) to solve the puzzles in the number of steps indicated. Trial and error would work for this puzzle, but allowing the students only a limited number of steps forces them to apply what they have learned about Malus' Law in the activity entitled Sunglasses and Polarizers (Sec. 3).

Students wear their sunglasses or look through their spinners. 


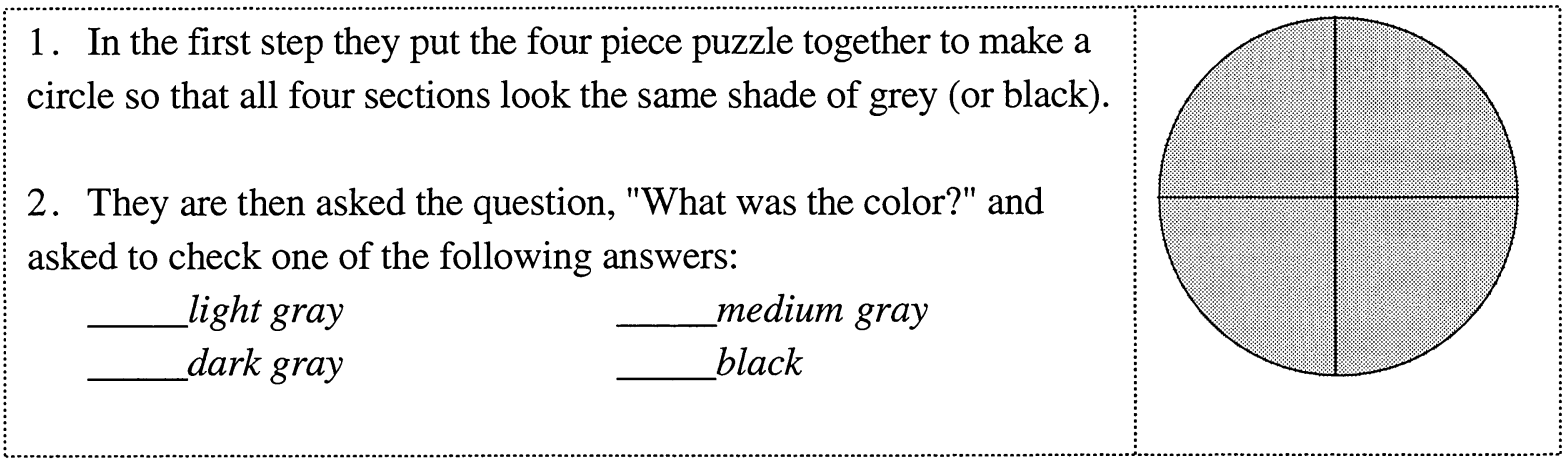

3. For the color they checked in step 2, they are asked to match the pair of polarizers below which best describes how the axes of the puzzle and their sunglasses/spinner are oriented.

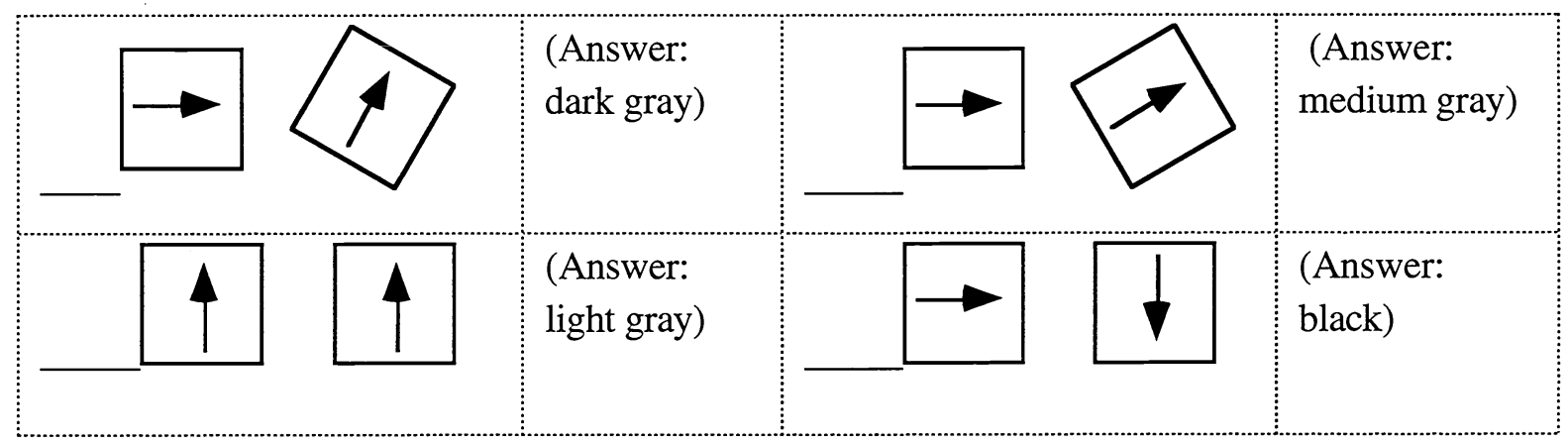

4. They are then asked to rotate the puzzle pieces so the whole circle is black.

(Spinner/ sunglass axis is perpendicular to that of the transmission axis of the puzzle.)

5. The students are then asked to exchange two pieces only and make the top half of the circle light gray and the bottom half black.

(Answer: interchanging the top two pieces will rotate both pieces by $90^{\circ}$ and make the top half light gray.)

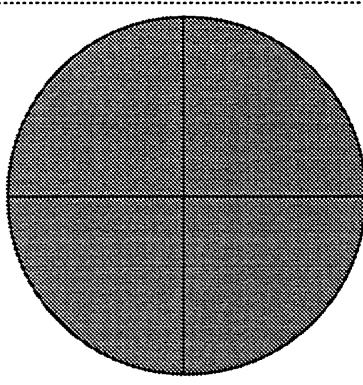


6. Next, they are asked to exchange only two pieces and make the pattern shown. (They may flip the pieces.)

(Answer: exchanging the two pieces on the right half of the circle rotates both of them by $90^{\circ}$ and makes the diagonal pieces light gray.)

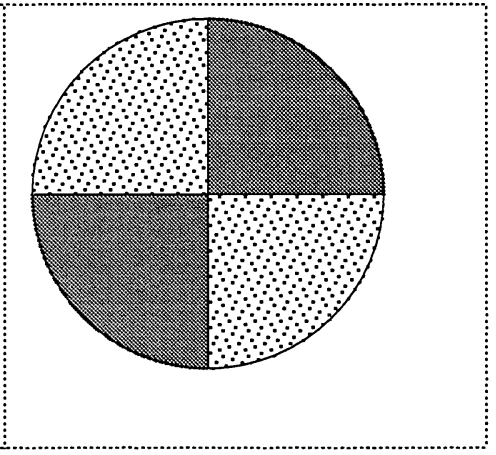

\subsection{The twelve piece puzzle}

This puzzle is the most challenging one of the lot. It requires some patience, and if a student can do it logically, they have a pretty good understanding of parallel and crossed polarizers!

Materials: A circle is cut from a linear polarizer, and then cut into twelve $30^{\circ}$ wedges: before separating the wedges, it is wise to mark the top side of each wedge with a piece of tape. Flipping the pieces over produces a larger number of equivalent pieces and makes the puzzle difficult to follow through. Students should be instructed not to flip pieces in this puzzle, and to always have the piece of tape on top as they do the puzzle. Students should wear their sunglasses, or hold the spinner in one position as they work.

1. The students start by arranging the pieces of the puzzle so that the whole circle is one color (gray).

2. They are then asked how the transmission axes of the pieces in the circle are oriented relative to their glasses (or spinner), and asked to draw the axes in the figure.

(Answer: the axes of all the pieces point in the same direction, parallel to the spinner when the color of the pieces is light gray.)

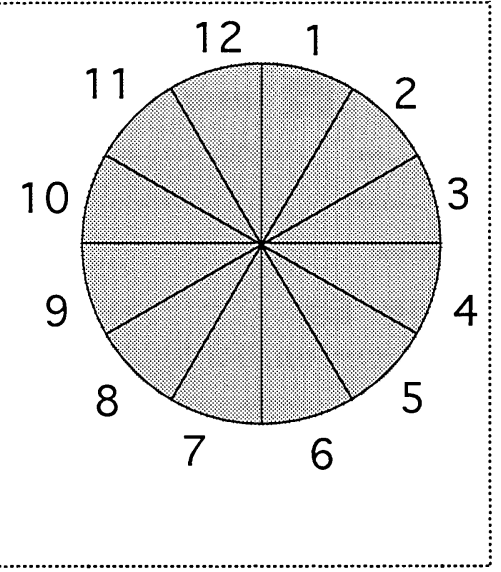


3. The students are then asked to move only four pieces and make the pattern shown (without flipping the pieces).

(Answer: move the piece numbered 3 in the figure above to the place occupied by piece 6,6 to 9,9 to 12 and 12 to 3 . Each of the pieces would have been rotated by $90^{\circ}$, making them dark compared to the gray of the pieces that were not moved.)

4. The students are asked to move the other eight pieces (in a simple sequential way), so they get the pattern shown in this figure.

(Answer: move piece 1 to the position occupied by piece 2, 2 to 4,4 to 5,5 to 7,7 to 8,8 to 10,10 to 11,11 to 1 . The pieces get rotated by either $30^{\circ}$ or $60^{\circ}$, and therefore look light gray or medium gray, respectively.)

An interesting variation on the last four activities (the spinning wheel, flashing wheel, four and twelve piece puzzles) is to glue cellophane to the back of the polarizer. When the pieces are placed with the cellophane side down on the light box, the puzzles appear shades of gray. When they are flipped over, they appear colored due to the birefringence in the cellophane.

In addition to these activities students made animated pictures using pre-fabricated polarmotion materials, some of which are wheels similar to those described (supplied by American Polarizer, Inc.).

In conclusion, we have presented several activities to introduce upper elementary students to concepts in linear polarization and qualitative aspects of Malus' Law. The activities were part of an extra curricular program to interest female students in Physics ${ }^{3}$. The activities outlined in this paper have been tested primarily on fifth and sixth grade female students, with considerable success. Students found all the activities interesting and well within their level of comprehension. Only one activity, the twelve piece puzzle, was perceived as challenging, but could be done with some patience. The total time taken by students to complete all activities described in this paper was about two and a half to three hours. 


\section{ACKNOWLEDGEMENTS}

We thank Michael L. Wallace for several helpful discussions. This work was supported by the National Science Foundation, Directorate for Education and Human Resources through the Model Projects for Women and Girls program, grant number HRD9253434.

\section{REFERENCES}

+ e-mail address for M. Chandrasekhar: phys3470@mizzou1.missouri.edu

* Present address: Department of Physics, University of Wisconsin, Madison WI.

1. While both electric and magnetic fields "wiggle" in a light wave, we do not go into this discussion at this point, since it usually confuses the students. For the precocious student who recalls that light is an electromagnetic wave, we discuss that one usually only worries about the direction in which the electric field wiggles, since the magnetic field always wiggles at right angles to it.

2. This explanation is strictly correct for a mechanical wave but not for an electromagnetic wave. Due to the induced electric field produced in a polarizer by the electromagnetic wave, the wave is transmitted when the direction of the electric field is perpendicular to the "slats", and not transmitted when it is parallel to the slats. At this age level, however, we do not enter into this discussion.

3. Activities in other areas of Optics and in Electricity and Magnetism, Sound, and Mechanics have also been developed as part of the program. For activity booklets contact the first author by letter or by e-mail at the address above. 